\title{
FACT - Calibration of Imaging Atmospheric Cherenkov Telescopes with Muon Rings
}

\author{
M. Nöthe, ${ }^{* a}$ M. L. Ahnen ${ }^{b}$, M. Balbo ${ }^{c}$, M. Bergmann $^{d}$, C. Bockermann ${ }^{e}$, A. Biland ${ }^{b}$, \\ T. Bretz ${ }^{b}$, K. A. Brügge ${ }^{a}$, J. Buss ${ }^{a}$, D. Dorner ${ }^{d}$, S. Einecke ${ }^{a}$, J. Freiwald $^{a}$, \\ C. Hempfling ${ }^{d}$, D. Hildebrand ${ }^{b}$, G. Hughes ${ }^{b}$, W. Lustermann ${ }^{b}$, K. Mannheim ${ }^{d}$, \\ K. Meier ${ }^{d}$, K. Morik ${ }^{e}$, S. Müller ${ }^{b}$, D. Neise ${ }^{b}$, A. Neronov $^{c}$, A.-K. Overkemping ${ }^{a}$,

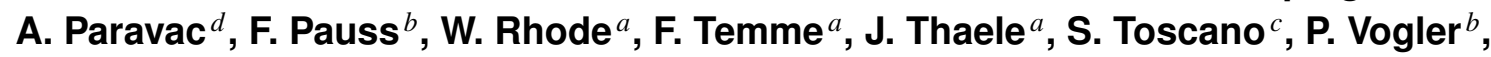 \\ R. Walter ${ }^{c}$, and A. Wilbert ${ }^{d}$ \\ Email: maximilian. noethedtu-dortmund.de
}

${ }^{a}$ TU Dortmund, Experimental Physics 5

Otto-Hahn-Str. 4, 44221 Dortmund, Germany

${ }^{b}$ ETH Zurich, Institute for Particle Physics

Otto-Stern-Weg 5, 8093 Zurich, Switzerland

${ }^{c}$ University of Geneva, ISDC Data Center for Astrophysics

Chemin d'Ecogia 16, 1290 Versoix, Switzerland

${ }^{d}$ Universität Würzburg, Institute for Theoretical Physics and Astrophysics

Emil-Fischer-Str. 31, 97074 Würzburg, Germany

${ }^{e}$ TU Dortmund, Lehrstuhl für künstliche Intelligenz.

Otto-Hahn-Str. 12, 44221 Dortmund, Germany

\begin{abstract}
The First G-APD Cherenkov Telescope (FACT) is an Imaging Air Cherenkov Telescope (IACT) located on the Canary Island of La Palma. Its goal is to provide long term monitoring of cosmic objects like Active Galactic Nuclei. FACT is the first IACT to use Silicon Photomultipliers instead of conventional Photo Multiplier Tubes. Therefore studying the detector properties is especially important.

Muon Rings are an event class that can offer valuable insight into the detector. These images are created by single muons that hit the mirror of the telescope. Due to their unique shape, one can use pattern recognition techniques like the Hough-Transform to identify these events.

The well-known properties of muon rings and their small photon arrival time distribution allow to estimate the time resolution, photon detection efficiency and optical Point-Spread-Function of the telescope.

In this contribution, the multivariate method using a random forest to extract muons from FACT data will be presented. Also the first results, including the time resolution of FACT and the impact of the mirror alignment in May 2014 on the ring width of muon events, are shown.
\end{abstract}

The 34th International Cosmic Ray Conference,

30 July - 6 August, 2015

The Hague, The Netherlands

\footnotetext{
*Corresponding author
} 


\section{Introduction}

FACT, the First G-APD Cherenkov Telescope, is an Imaging Air Cherenkov Telescope, located on the Canary Island of La Palma. FACT is the first IACT that uses Silicon Photomultipliers (SiPMs) instead of conventional Photomultiplier Tubes to detect Cherenkov photons, produced by charged, secondary particles in extensive air showers. For more information, see [1, 2].

Thanks to the robustness of SiPMs, FACT can observe even during full moon, making it an ideal instrument for long term monitoring of TeV gamma ray sources. The FACT camera is comprised of 1440 SiPM pixels. The reflector of FACT is made of 30 hexagonal mirros with a total reflective area of $9.51 \mathrm{~m}^{2}$. The focal length is $4.9 \mathrm{~m}$ and the resulting field of view is $4.5^{\circ}$. These mirros were aligned in the Davies-Cotton-Orientation until May 2014, when the mirror orientation was changed to a hybrid between Davies-Cotton- and a parabola-orientation. [9, 10]

Since SIPMs are a novel technology in Cherenkov Astronomy, studying the properties of these sensors is especially important. Muon ring images provide a valuable class of events to estimate the performance of the complete detector, from the mirrors to the analysing software.

\section{Simulation using CORSIKA}

For the training of a supervised machine learning algorithm labeled data is needed. This training data is created using CORSIKA [8] to simulate muons and proton induced extensive air showers. The CORSIKA output is then fed into the software CERES [5], which takes care of the reflector and camera simulation. For the signal class, muons are simulated for energies of $10 \mathrm{GeV}$ to $1 \mathrm{TeV}$ using a spectral index of -2.7 . They are inserted at an altitude of $2500 \mathrm{~m}, 300 \mathrm{~m}$ above the telescope, in zenith direction. To produce only complete ring images that are contained in the camera, the muons are simulated with an impact parameter of less than the mirror diameter and with an angle to the optical axis of less than $2^{\circ}$. For the background data, the same proton dataset as described in proceeding [12] is used.

\section{Preprocessing using FACT-Tools}

The preprocessing of the raw data taken by the telescope or created by the simulation software is done using the software framework FACT-Tools [3]. FACT-Tools is an extension of the streams framework, developed by the Artificial Intelligence Group, Faculty for Informatics at TU Dortmund[4]. The following steps are done in this software:

1. Calibration of the voltage curves for each pixel.

2. Extraction of number of photons and their arrival time.

3. Image cleaning to select a subset of pixels belonging to the physics event.

4. Feature generation, e.g. the standard Hillas parameters.

The software allows for rapid prototyping thanks to its easy to use xml interface and well constructed event viewer. For more information see, the proceeding [6]. 


\section{Feature generation using the Hough-Transform}

One of the most important aspects in Machine-Learning is a proper feature generation. The standard Hillas parameters, especially Width, Length and Size, show some discrimination power between proton and muon events. Timing properties, e.g. the standard deviation of the photon arrivalTimes, show discrimination between the two event classes. To further improve the discrimination, features are created, which are based on on a shape recognition algorithm looking for rings in the camera images.

A well known and widely used shape recognition algorithm is the so called Hough Transform, first introduced by Paul Hough in 1962 [7]. The algorithm was used to generate features for muon event recognition by Jonathan Tyler for the VERITAS Collaboration [13].

The image data is transformed into a discrete parameter space. For the case of muon ring images the photoncharge of a pixel at position $\left(x_{\text {pix }}, y_{\text {pix }}\right)$ is transformed into the total photoncharge of a ring with radius $R$ and center coordinates $(x, y)$.

The parameter space is discretized using a step width of $3.3 \mathrm{~mm}$ for $R$ and $10 \mathrm{~mm}$ for $x$ and $y$. The limits of the parameter space are $[40 \mathrm{~mm}, 110 \mathrm{~mm}]$ for $R$ and $[-300 \mathrm{~mm}, 300 \mathrm{~mm}]$ for $x$ and $y$.

For every possible combination of $x, y$, and $R$, the photoncharge of each pixel belonging to this ring is summed up. A pixel belongs to the ring, if it is not farther away from the ring than the pixel diameter. The resulting 3-dimensional matrix $H$ is called the hough accumulator.
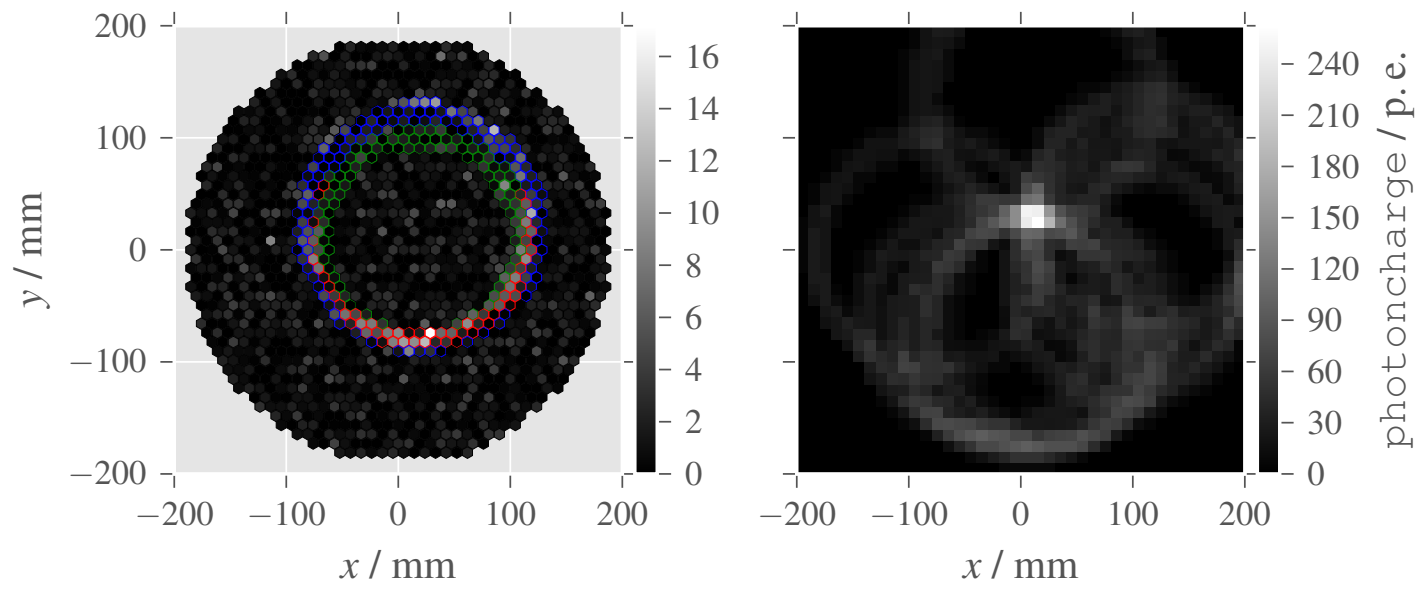

Figure 1: Left: Camera image of a simulated muon event with reconstructed number of photons for each pixel. Right: Transformation result for a simulated muon ring. The $x, y$-plane of the $H$ matrix is shown for the slice of $R$ with the maximal value. The blue and green pixels represent the two rings with the maximal photoncharge, the intersection is marked in red.

The transformation result for a simulated muon ring is shown in Figure 1. A very prominent peak is visible at the most likely position of the ring center. The two highest counting rings, shown in green and blue, are close to each other.

The transformation result for a proton shower, see Figure 2, looks quite different. There is no clear maximum but a ring of larger values and the two highest entries are on opposite sides of the ring. These properties lead to two parameters describing the matrix: 

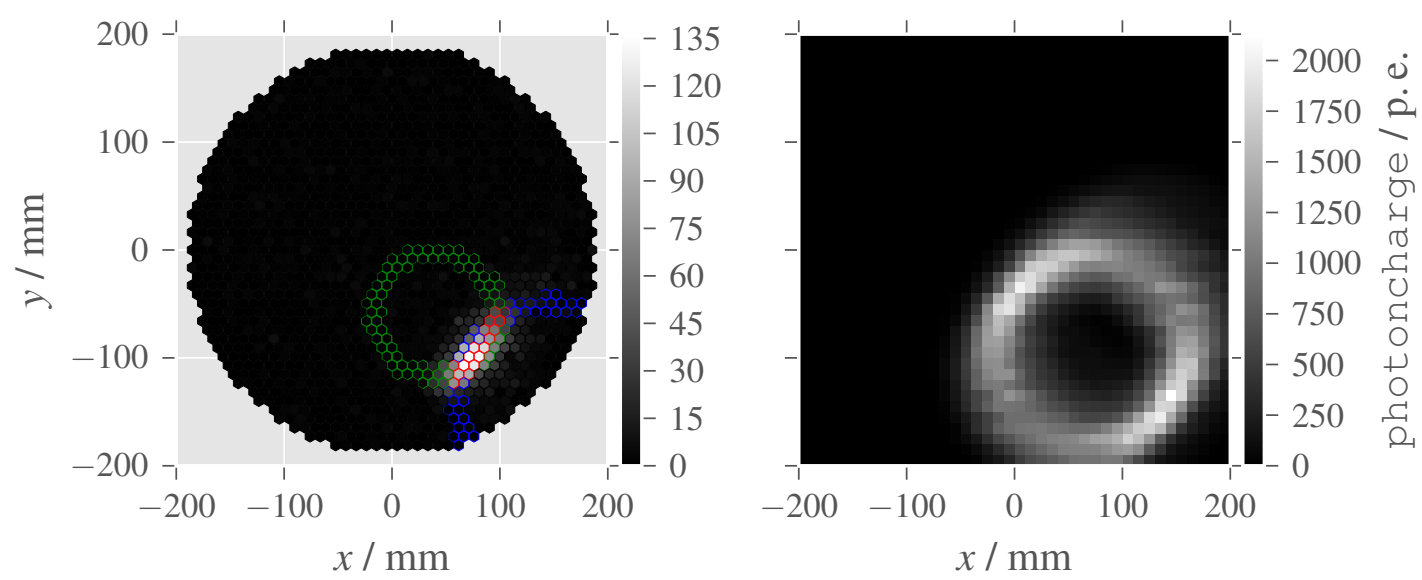

Figure 2: Left: Camera image for a simulated proton event with reconstructed number of photons for each pixel. Right: Transformation result for a simulated proton shower. The $x, y$-plane of the $H$ matrix is shown for the slice of $R$ with the maximal value. The blue and green pixels represent the two rings with the maximal photoncharge, the intersection is marked in red.

- The houghPeakness, defined as the maximum value of $H$ divided by the mean value for all non-zero entries.

- The houghDistance, defined as the sum of the euclidean distances in parameter space between the three best fitting rings.

Both parameters were proposed by J. Tyler in [13]. Additionally two more parameters are calculated from the result of the Hough-Transform:

- The houghCleaningPercentage, defined as the number of pixels that survived cleaning and are on the best fitting ring divided by the total number of pixels that survived cleaning.

- The houghRingPercentage, defined as the number pixels that survived cleaning and belong to the ring divided by the total number of pixels belonging to the ring.

\section{Multivariate separation using scikit-learn}

The separation between muon ring images and background events, mainly proton induced air showers, is done using the Python machine learning framework sklearn [11].

A set of 50000 proton and 50000 muon events is used for training and evaluation.

In total 42 features, including the standard Hillas parameters, the Hough features described in Section 4 and higher order moments of the photoncharge and arrivaltime distributions are fed into the algorithm.

A 10-fold cross validation is done to estimate the performance of the classifier. The area under the ROC-curve (roc_auc) is used as performance criterion for the classifier. A perfect classification would result in roc_auc $=1$. For equally sized signal and background data sets, the worst case is roc_auc $=0.5$. This value is used for the optimization of the parameters of the random forest, e. g. the number of features in each node or the maximum depth of the trees. 
After selecting the classifier with the highest roc_auc the recall and precision of the classification is evaluated. On the training set,

$$
\text { roc_auc }=0.994 \pm 0.001
$$

was reached with 200 trees, 13 features per node and a maximum depth of 14 .

Events with a confidence $>0.93$ are classified as muons. This cut was choosen, because it delivers a high precision of $0.987 \pm 0.002$ at a reasonable recall of $0.881 \pm 0.004$.

\section{Muon Ring Width as Indicator of the Point-Spread-Function}

The width of the muon ring images is dominated by the spatial resolution of the telescope. The convolved light distribution is folded with the reflector geometry. The convolution kernel is called Point-Spread-Function (PSF). It represents the image of a point source in the camera plane. To get an estimate of the PSF, a gaussian distribution is fit to each ring image. The standard deviation of the gaussian should be an indicator for the Point-Spread-Function of the Telescope.

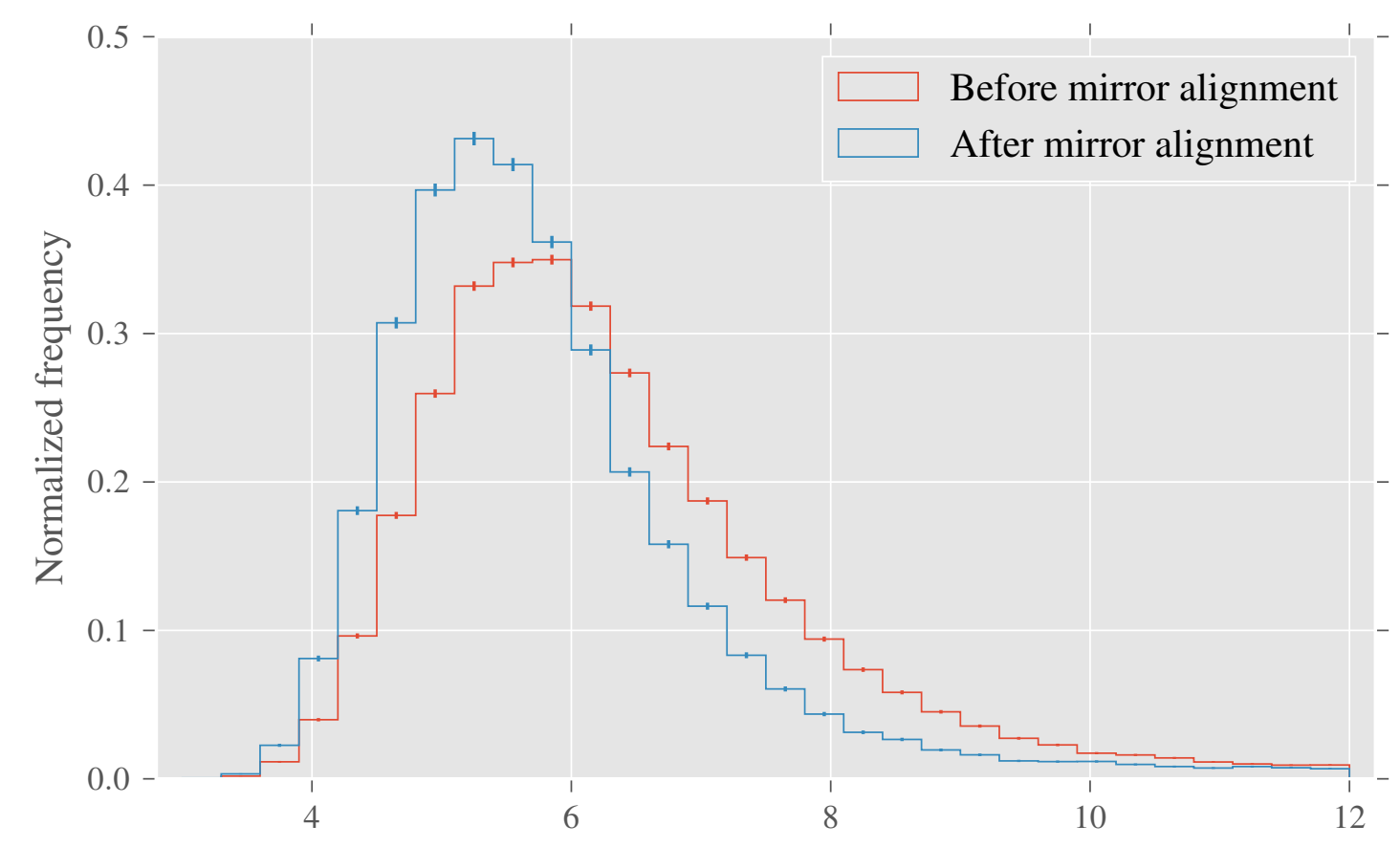

Std. dev. of radial light distribution / $\mathrm{mm}$

Figure 3: Distributions of the muon ring width before and after the mirror alignment in May 2014. The areas of the histograms are normalized to 1 .

The Maximum-Likelihood-Fit is performed using following negative Log-Likelihood-Function:

$$
-\ln (\mathscr{L}(x, y, R, \sigma))=\sum_{\text {Pixel } i}\left(\ln \sigma+\frac{\left(\sqrt{\left(x_{i}-x\right)^{2}+\left(y_{i}-y\right)^{2}}-R\right)^{2}}{\sigma^{2}}\right) \cdot \text { photoncharge }_{i}
$$


Where $(x, y)$ is the center of the ring, $R$ its Radius, $\sigma$ is the standard deviation of the gaussian and $x_{i}$, $y_{i}$ are the coordinates of Pixel $i$.

In May 2014, a mirror alignment of FACT was performed. The distribution of $\sigma$ for the datasets before and after the alignment are shown in figure 3. A clear improvement due to the mirror alignment is visible.

\section{Time Resolution}

The arrival time distribution of the muon ring photons at the telescope reflector is neglectable compared to the time resolution of the telescope. Therefore the distribution of the reconstructed arrival times is a measurement of the time resolution. This analysis includes the effects of every aspect of the analysis chain, including the timing properties of the reflector, see [9], the SIPMs and the readout electronics, as well as the calibration and arrival time extraction used in FACT-Tools.

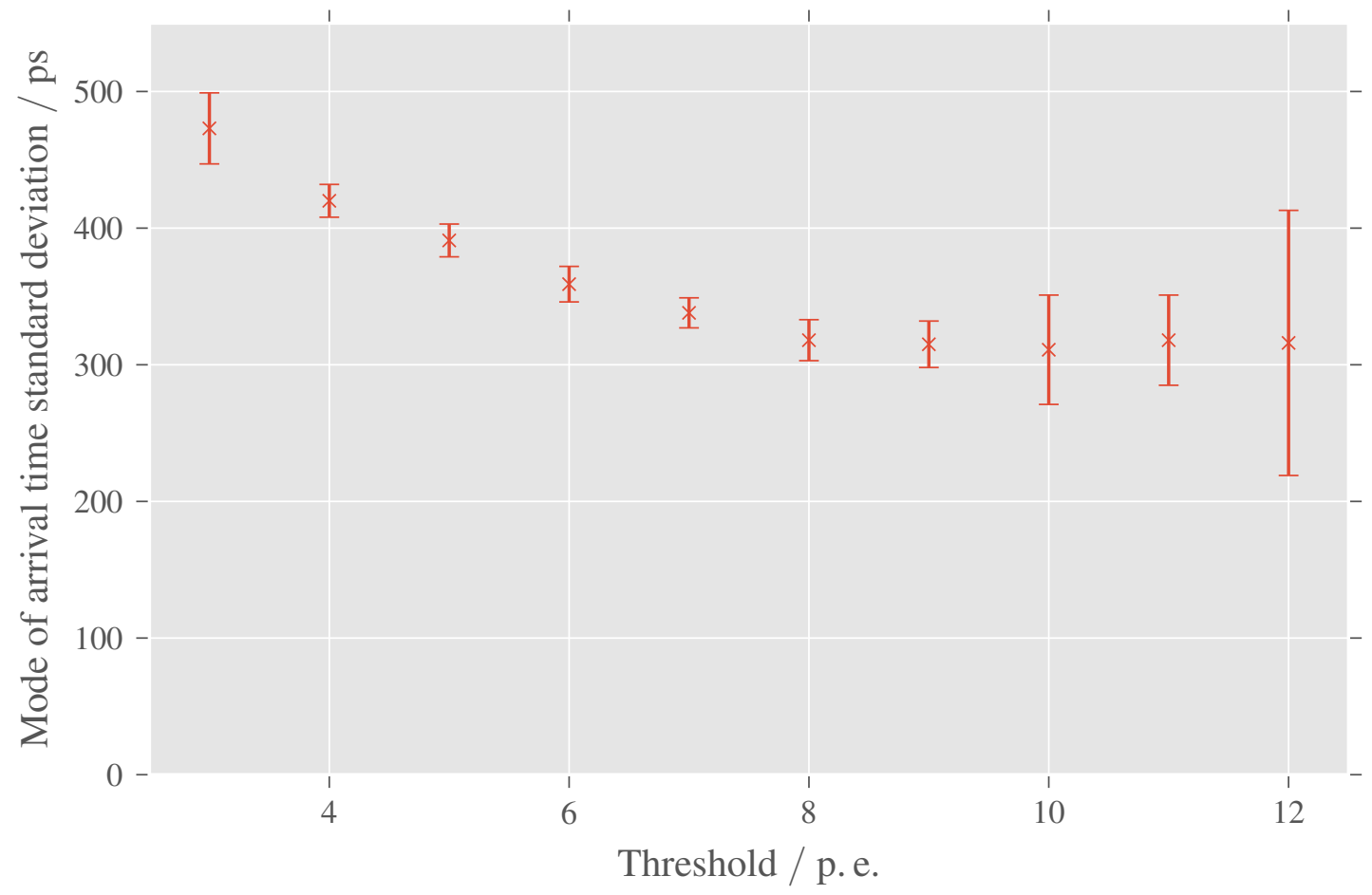

Figure 4: Mode of the standard deviation of photon arrival times in muon ring images versus the threshold applied to the photoncharge of the pixels. Errors are calculated via a 200-fold bootstrap validation. The distribution converges for thresholds $>8 \mathrm{p}$. e.

In the following the cleaned images, which were identified as muons using the methods described above, are used to calculate the time resolution of FACT with respect to a lower limit for the photon count in each pixel. Additionally, only pixels are considered, which are less than $10 \mathrm{~mm}$ away from the best fitting ring of the Hough transform.

For larger pulses, induced by more photons, arrival time extractors, which are based on finding the rising edge of the voltage time series, should perform better. Low photon pixels can also be dominated by night sky background photons, which are uniformly distributed in time. 
To investiage the behaviour of the time resolution, only pixels with a photon count above a certain threshold are considered for calculating the standard deviation in each event. Calculating a standard deviation based on just a few values is not meaningful, therefore only events where more than 5 pixels entered into the calculation are considered. The mode of the distributions for different thresholds is shown in figure 4 . The value converges to about $320 \mathrm{ps}$ for thresholds larger than $8 \mathrm{p}$. e. The distribution of the standard deviations for a threshold of 8 p. e. is shown in figure 5 .

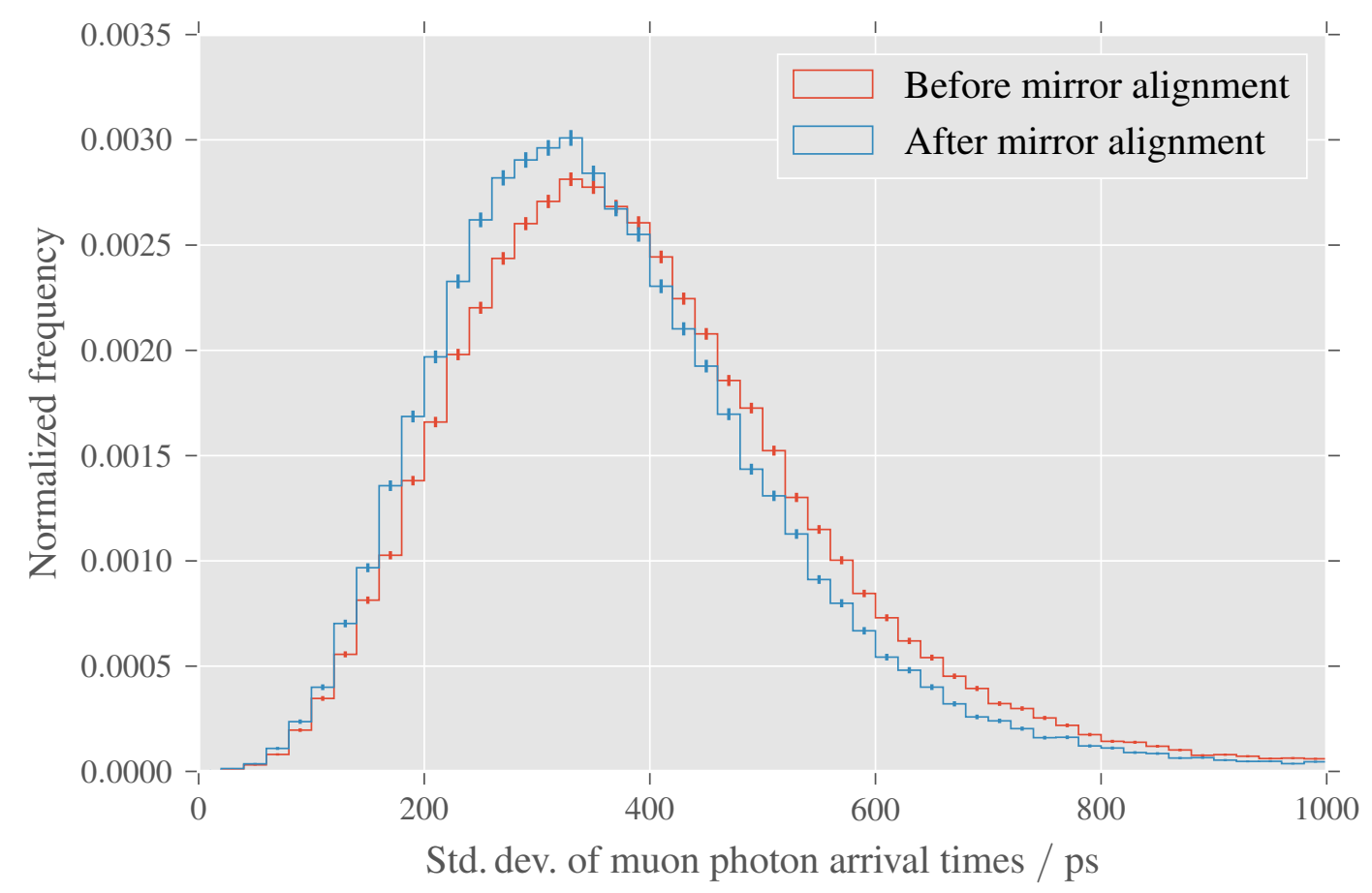

Figure 5: Distribution of the standard deviation of photon arrival times in muon ring images for a threshold of 8 p. e. The data taken before the mirror alignment is show in red, data taken after the alignment in blue. The areas of the histograms are normalized to 1.

As the mirror alignment changed also the timing properties of the reflector, the data is again split into parts before and after the mirror alignment. The data shows a small improvement of the time resolution because of the mirror alignment.

\section{Conclusions}

The multivariate extraction of muon ring images from the FACT-data, using features derived from a Hough-Transform, performs very well. The extracted events were used to calculate the time resolution of the FACT-Telescope for several thresholds in the number of extracted photons. The resolution is in $\mathscr{O}(100 \mathrm{ps})$, with the mode of the distribution around $320 \mathrm{ps}$ for thresholds above 8 p.e.

The ring width, derived from gaussian fits to the image data, shows a clear improvement of the PSF due to the mirror alignment in May 2014. This property of muon rings could be used for monitoring the telescope and deciding whether a new mirror alignment is needed. 
Acknowledgment The important contributions from ETH Zurich grants ETH-10.08-2 and ETH-27.12-1 as well as the funding by the German BMBF (Verbundforschung Astro- und Astroteilchenphysik) and HAP (Helmoltz Alliance for Astroparticle Physics) are gratefully acknowledged. We are thankful for the very valuable contributions from E. Lorenz, D. Renker and G. Viertel during the early phase of the project. We thank the Instituto de Astrofisica de Canarias allowing us to operate the telescope at the Observatorio del Roque de los Muchachos in La Palma, the Max-Planck-Institut für Physik for providing us with the mount of the former HEGRA CT 3 telescope, and the MAGIC collaboration for their support. Part of this work is supported by Deutsche Forschungsgemeinschaft (DFG) within the Collaborative Research Center SFB 876 "Providing Information by Research-Constrained Analysis", project C3.

\section{References}

[1] H. Anderhub et al. Design and operation of FACT - the first G-APD Cherenkov telescope. In: JINST 8.06 (2013), P06008.

[2] A. Biland et al. Calibration and performance of the photon sensor response of FACT - the first G-APD Cherenkov telescope. In: JINST 9.10 (2014), P10012.

[3] C. Bockermann et al. FACT-Tools Bitbucket-Repository. URL: https : / / bitbucket . org/cbockermann/fact-tools.

[4] C. Bockermann and H. Blom. The Streams Framework. Tech. rep. 5. TU Dortmund, Dec. 2012.

[5] T. Bretz, D. Dorner, et al. MARS - CheObs ed.: A flexible software framework for future Cherenkov telescopes. In: Proceedings of the 11th ICATPP Conference. 2009.

[6] K. Brügge et al. FACT-Tools: Streamed Real-Time Data Analysis. In: these proceedings. 1135. 2015.

[7] P. E. Hart. How the Hough Transform Was Invented. In: IEEE Signal Processing Magazine 26 (2009), pp. 18-22.

[8] D. Heck et al. CORSIKA: A Monte Carlo Code to Simulate Extensive Air Showers," Report FZKA 6019, Forschungszentrum Karlsruhe. Tech. rep. 1998.

[9] S. Müller. Clear Sight in Cherenkov-Astronomy. Investigations of Reflector Geometry and Mirror Alignment for the FACT-Telescope. MA thesis. TU Dortmund, 2014.

[10] S. Müller et al. FACT - Novel mirror alignment using Bokeh and enhancement of the VERITAS SCCAN alignment method. In: these proceedings. 523. 2015.

[11] F. Pedregosa et al. Scikit-learn: Machine Learning in Python. In: Journal of Machine Learning Research 12 (2011), pp. 2825-2830.

[12] F. Temme et al. FACT - First Energy Spectrum from a SiPM Cherenkov Telescope. In: these proceedings. 174. 2015.

[13] J. Tyler. Muon identification with VERITAS using the Hough Transform. MA thesis. McGill University Montreal, 2011. 